\title{
TSaT-MUSIC: a novel algorithm for rapid and accurate ultrasonic 3D localization
}

\author{
Kyohei Mizutani ${ }^{1 *}$, Toshio Ito ${ }^{1}$, Masanori Sugimoto ${ }^{1}$ and Hiromichi Hashizume ${ }^{2}$
}

\begin{abstract}
We describe a fast and accurate indoor localization technique using the multiple signal classification (MUSIC) algorithm. The MUSIC algorithm is known as a high-resolution method for estimating directions of arrival (DOAs) or propagation delays. A critical problem in using the MUSIC algorithm for localization is its computational complexity. Therefore, we devised a novel algorithm called Time Space additional Temporal-MUSIC, which can rapidly and simultaneously identify DOAs and delays of mul-ticarrier ultrasonic waves from transmitters. Computer simulations have proved that the computation time of the proposed algorithm is almost constant in spite of increasing numbers of incoming waves and is faster than that of existing methods based on the MUSIC algorithm. The robustness of the proposed algorithm is discussed through simulations. Experiments in real environments showed that the standard deviation of position estimations in 3D space is less than $10 \mathrm{~mm}$, which is satisfactory for indoor localization.
\end{abstract}

Keywords: MUSIC-Algorithm, Ultrasound, Adaptive Array, Computational Complexity, 3D Localization, DOA-Delay Estimation

\section{Introduction}

In recent years, localization techniques have attracted considerable attention in ubiquitous computing communities. There have been many studies on localizing objects by using ultrasonic signals; for example, indoor positioning [1-3] or robotics [4,5]. There are several requirements for localization techniques, including accuracy, robustness, and ease of deployment.

We propose a new localization technique using the Time Space additional Temporal MUSIC (TSaTMUSIC) algorithm, a variant of the MUltiple SIgnal Classification (MUSIC) algorithm [6]. The principle advantage of the TSaT-MUSIC algorithm is its low computational complexity compared with other variants of the MUSIC algorithm.

The MUSIC algorithm is a well-known method for direction of arrival (DOA) or propagation delay estimations. As the algorithm conducts null steering of incoming waves, it shows higher resolution than the main beam steering methods such as the delay and sum

\footnotetext{
* Correspondence: mizutani@itl.t.u-tokyo.ac.jp

${ }^{1}$ Department of Electrical Engineering and Information Systems, School of Engineering, University of Tokyo 7-3-1 Hongo, Bunkyo-ku, Tokyo, 113-8656, Japan

Full list of author information is available at the end of the article
}

beamforming methods. There have been many studies on 3D localization using MUSIC algorithm; for example, [7]. The Spatial-MUSIC (S-MUSIC) algorithm, which is just called the MUSIC algorithm, is used for DOA estimations. Another variant of the MUSIC algorithm called Temporal-MUSIC (T-MUSIC) offers propagation delay estimates. By using these algorithms, we can identify either the DOA or the delay but not both simultaneously.

To estimate the DOA and delay simultaneously using these algorithms, two main famous approaches have been proposed. The first applies the MUSIC algorithm to spatial- and frequency-domain data at the same time; for example, 2D-MUSIC [8], 2D-TDM MUSIC [9], and JADE-MUSIC [10]. This approach conducts a 2D search of angle and time. Thus, its computational complexity becomes very large. The second approach integrates other DOA or delay estimation methods with the MUSIC algorithm, such as [11]. For instance, TSTMUSIC [12] uses beamforming and temporal filtering methods. Compared with the first approach, the second approach has less computational complexity. However, it still requires high computation times, because the computational complexity increases in proportion to the number of incoming waves. 
Our proposed algorithm can estimate DOA and delay values simultaneously in an entirely different manner from the existing approaches. The procedure of the TSaT-MUSIC algorithm is as follows. First, sets of DOA and delay values are estimated using the S-MUSIC and T-MUSIC algorithms, respectively. Next, the true pairs of DOA and delay values are decided by applying the TMUSIC algorithm at a sensor different from the sensor used in the first step. As a result, we can estimate DOAs and delays with only three MUSIC algorithm executions. The original TSaT-MUSIC algorithm is for DOA-delay estimation in a $2 \mathrm{D}$ space and can easily be extended to a localization method in a 3D space.

One advantageous point of MUSIC algorithms used for $3 \mathrm{D}$ localization is that we can design a compact receiver array. In this study, a small L-shaped receiver array (about $36 \mathrm{~mm} \times 36 \mathrm{~mm}$ ) is implemented to evaluate the performance of the TSaT-algorithm for 3D localization.

The objective of this paper is to prove that the proposed method reduces the computational complexity of sound source localization and still retains the satisfactory level of accuracy. Thus, we conduct comparative evaluations using the TST-MUSIC algorithm, which is one of the fastest and most accurate localization methods using MUSIC algorithms.

In this paper, a brief introduction of the MUSIC algorithm is first presented. then, the proposed algorithm and its 3D localization method are explained. Subsequently, the results of computer simulations, and experiments in real environments using the TSaT-MUSIC algorithm are reported.

\section{The MUSIC algorithm}

\subsection{Data model}

First, we define the data model that is adopted in this paper. Figure 1 shows the configuration of a sensor array. We assume that the transmitted signal consists of multicarrier ultrasonic waves and that a linear sensor

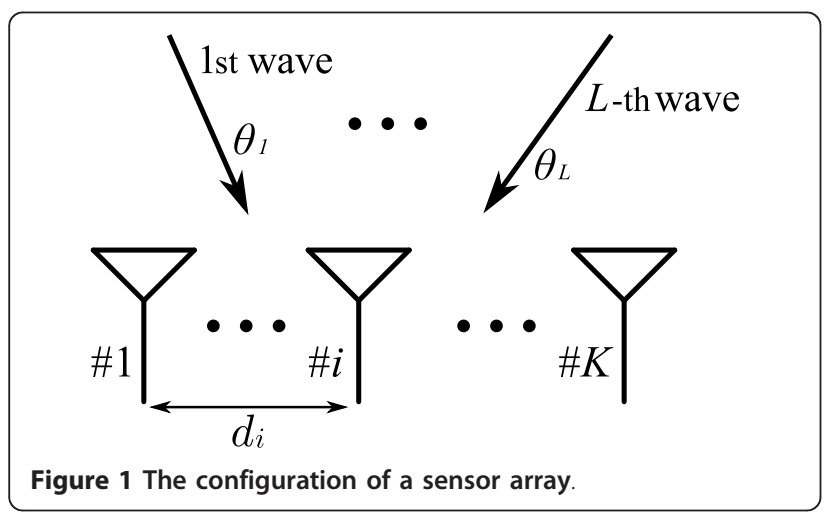

array is used. The numbers of sensors and frequencies are defined as $K$ and $M$, respectively. By using the Fourier transform for received signals at each sensor, we obtain a received data matrix $X$. The dimension of $X$ is $K \times M$. We define the received data for the mth frequency at the $k$ th sensor as $x_{k, m}$, so $X$ can be written as:

$$
X=\left[\begin{array}{ccc}
x_{1,1} & \cdots & x_{1, M} \\
\vdots & \ddots & \vdots \\
x_{K, 1} & \cdots & x_{K, M}
\end{array}\right] .
$$

Then, two received data vectors can be defined. A vector $S_{m}(m=1,2, \ldots, M)$ is the received data vector at the mth frequency, and a vector $\boldsymbol{T}_{\boldsymbol{k}}(k=1,2, \ldots, K)$ is the data received at the $k$ th sensor. Thus, $S_{m}$ and $\boldsymbol{T}_{\boldsymbol{k}}$ can be expressed as:

$$
\begin{aligned}
S_{m} & =\left[x_{1, m}, x_{2, m}, \ldots, x_{K, m}\right]^{T} \\
T_{k} & =\left[x_{k, 1}, x_{k, 2}, \ldots, x_{K, M}\right]^{T},
\end{aligned}
$$

where the superscript $[\cdot]^{T}$ denotes the matrix transpose operation. Next, we introduce the mode vector $\boldsymbol{a}_{\boldsymbol{m}}$ $\left(\theta_{l}\right)(m=1,2, \ldots, M)$, where $\theta_{1}$ is the angle of the $l$ th wave. $a_{m}\left(\theta_{l}\right)$ is defined as:

$$
a_{m}\left(\theta_{l}\right)=\left[\exp \left(j \Psi_{m, 1}\left(\theta_{l}\right)\right), \ldots, \exp \left(j \Psi_{m, K}\left(\theta_{l}\right)\right)\right]^{T} .
$$

$\Psi_{m, k}\left(\theta_{l}\right)(k=1,2, \ldots, K)$ is the phase of the $l$ th wave of the $m$ th frequency at the $k$ th sensor and is expressed as:

$$
\Psi_{m, k}\left(\theta_{l}\right)=-2 \pi f_{m} \frac{d_{k} \sin \theta_{l}}{c},
$$

where $c$ is the velocity of sound, $f_{m}$ is the $m$ th frequency, and $d_{k}$ is the distance between the $k$ th sensor and the 1st sensor (as shown in Figure 1). Assuming that the receiving waves are plane waves, $S_{m}$ can be written as:

$$
\begin{gathered}
S_{m}=A_{m} F+N, \\
\left\{\begin{array}{l}
A_{m}=\left[a_{m}\left(\theta_{1}\right), \ldots, a_{m}\left(\theta_{L}\right)\right], \\
F=\left[F_{1}, \ldots, F_{L}\right]^{T}
\end{array}\right.
\end{gathered}
$$

where $F_{l}$ is the complex amplitude of the $l$ th wave, and $N$ is the Gaussian noise vector with zero means and equal variances $\sigma^{2}$.

Similarly, we introduce a new mode vector $\boldsymbol{g}_{\boldsymbol{k}}\left(\tau_{l}\right)(k=$ $1,2, \ldots, K)$, where $\tau_{l}$ is the propagation delay time of the lth wave. $g_{\boldsymbol{k}}$ is defined as:

$$
g_{k}\left(\tau_{l}\right)=\left[\exp \left(-j 2 \pi f_{1} \tau_{l}\right), \ldots, \exp \left(-j 2 \pi f_{M} \tau_{l}\right)\right]^{T} .
$$

By using $g_{k}, T_{k}$ can be written as:

$$
T_{k}=G_{k} F+N,
$$


where $\boldsymbol{G}_{\boldsymbol{k}}$ is:

$$
G_{k}=\left[g_{k}\left(\tau_{1}\right), \ldots, g_{k}\left(\tau_{L}\right)\right]
$$

\subsection{The S-MUSIC algorithm}

The S-MUSIC algorithm is for DOA estimation. This algorithm is used with single-frequency signals. By Equation (1), the correlation matrix calculated using $\mathbf{S m}$ is given as:

$$
\begin{aligned}
R s s & =E\left[S_{m} S_{m}{ }^{H}\right] \\
& =A_{m} E\left[F F^{H}\right] A_{m}{ }^{H}+E\left[N N^{H}\right] \\
& =A_{m} \alpha A_{m}{ }^{H}+\sigma^{2} I\left(\alpha \equiv E\left[F F^{H}\right]\right),
\end{aligned}
$$

where the superscript $[\cdot]^{H}$ denotes the Hermitian operation. The eigenvectors of Rss are the orthogonal direct sum of the signal subspace and the noise subspace. Assuming that the incoming waves are incoherent and that the value of $L$ is smaller than that of $K$, we can derive the MUSIC spectrum by using the eigenvectors $\boldsymbol{u}_{\boldsymbol{i}}(i=L+1, \ldots, K)$ that span the noise subspace of $\boldsymbol{R} \boldsymbol{s s}$. The MUSIC spectrum can be written as:

$$
P s(\theta)=\frac{a_{m}{ }^{H}(\theta) a_{m}(\theta)}{a_{m}{ }^{H}(\theta) U U^{H} a_{m}(\theta)}\left(U \equiv\left[u_{L+1}, \ldots, u_{K}\right]\right) .
$$

The DOAs can be obtained as the peak values of $P_{s}(\theta)$ by changing the angle $\theta$. As can be seen in Equation (3), the number of incoming waves $L$ is given. If $L$ is unknown, Akaike Information Criteria (AIC) or Minimum Description Length (MDL) [13] can be used to estimate $L$. When the incoming waves are coherent, the S-MUSIC Algorithm does not work properly. Therefore, spatial smoothing preprocessing (SSP) [14] is used to suppress the coherence.

\subsection{The T-MUSIC algorithm}

The T-MUSIC algorithm is for propagation delay estimations. It differs from the S-MUSIC algorithm in that the T-MUSIC algorithm uses only one sensor and multiple-frequency waves. We therefore use a received data vector $\boldsymbol{T}_{\boldsymbol{k}}$.

Considering the Equation (2), the form of this equation corresponds to that of Equation (1). Thus, we can derive the MUSIC spectrum of propagation delays $\operatorname{Pt}(\tau)$ in the same way as in the S-MUSIC algorithm. When the eigenvectors of the correlation matrix calculated using $\boldsymbol{T}_{\boldsymbol{k}}$ are defined as $\boldsymbol{v}_{\boldsymbol{i}}(i=1,2, \ldots, M), \operatorname{Pt}(\tau)$ can be described as:

$$
\operatorname{Pt}(\tau)=\frac{g_{k}{ }^{H}(\theta) g_{k}(\theta)}{g_{k}{ }^{H}(\theta) V V^{H} g_{k}(\theta)}\left(V \equiv\left[v_{L+1}, \ldots, v_{M}\right]\right) .
$$

The propagation delays are obtained by finding the peak values of $\operatorname{Pt}(\tau)$ in the same way as in the S-MUSIC algorithm.

When the bandwidth of the multicarrier waves is $f_{d}$, the T-MUSIC algorithm can estimate a delay time to an accuracy of $1 / f_{d}$.

\section{The TSaT-MUSIC algorithm}

\subsection{Principle}

By applying the T-MUSIC and S-MUSIC algorithms to $L$ incoming waves at sensor $\mathrm{A}$ in the sensor array their DOA and propagation delay values are described as $\left(\theta_{1}\right.$, $\left.\theta_{2}, \ldots, \theta_{\mathrm{L}}\right)$ and $\left(\tau_{1}, \tau_{2}, \ldots, \tau_{L}\right)$ as shown in Figure 2. By applying T-MUSIC again at sensor B in the same sensor array, propagation delays can be estimated as $\left(D_{1}, D_{2}, \ldots\right.$, $\left.D_{L}\right)$. The path length of the $l$ th incoming wave arriving at sensor $\mathrm{A}$ is $d \sin \theta_{l} / c$ longer than that of the wave arriving at sensor $\mathrm{B}$, where $d$ is the distance between the two sensors. Therefore, we can plot $L^{2}$ points as possible DOA-delay pairs in the $(d \sin \theta, c \tau)$ space. These points are called "candidate points". Here, the following equation must be fulfilled:

$$
c \tau_{l}-d \sin \theta_{l}=c D_{l}(l=1,2, \ldots, L) .
$$

We define the Equation (5) drawn in the $(d \sin \theta, c \tau)$ space as "path difference lines". Theoretically, there can be only one point that represents a correct DOA-delay pair on each line. In the situation shown in Figure 3, for example, the pair of DOA and delay values are estimated as $\left(\theta_{1}, \tau_{2}\right),\left(\theta_{2}, \tau_{3}\right),\left(\theta_{3}, \tau_{1}\right)$.

In real environments, however, the correct point is not always on the line because of noise. Hence, we calculate the distance $\operatorname{dist}(i, j, l)$ between each candidate point $(d \sin$ $\left.\theta_{i}, c \tau_{j}\right)$ and the path different line $c \tau-d \sin \theta=c D_{l}$ as:

$$
\operatorname{dist}(i, j, l)=\frac{1}{\sqrt{2}}\left|c \tau_{j}-d \sin \theta_{i}-c D_{l}\right| .
$$

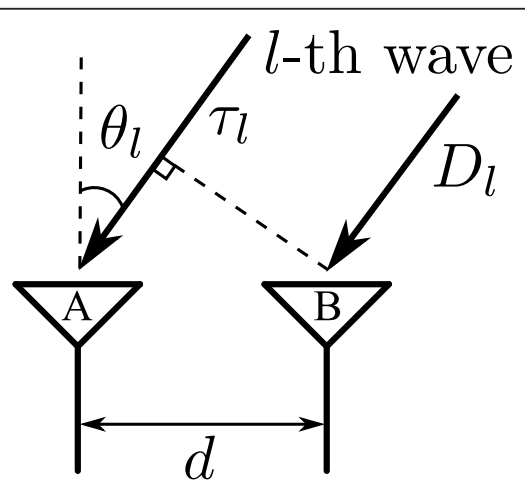

Figure 2 Relation between two ultrasonic receivers. 


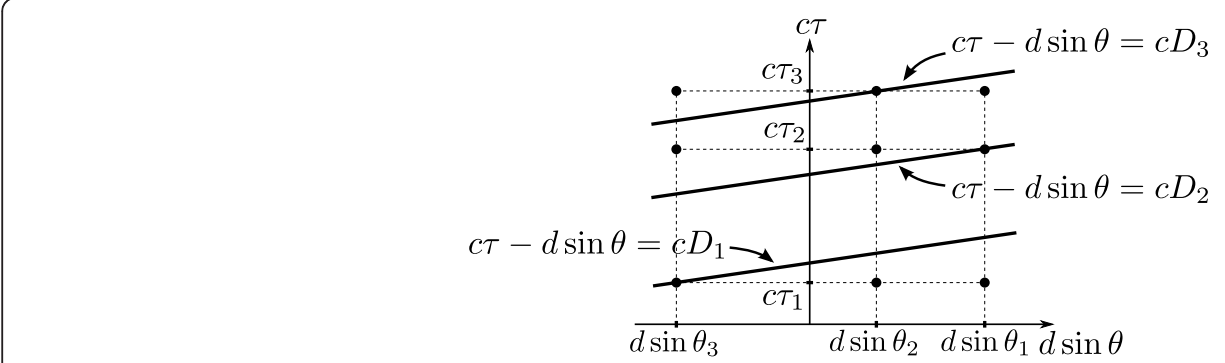

Figure 3 An example of $d \sin \theta-c \tau$ plots.

Then, the points with the minimum distance are selected as true points.

\subsection{D localization using the TSaT-MUSIC algorithm}

The TSaT-MUSIC algorithm allows us to simultaneously obtain the DOA and delay values in a 2D space. By using an L-shaped ultrasonic sensor array as shown in Figure 4, TSaT-MUSIC can be extended to a 3D localization algorithm.

We can estimate two angles, $\theta_{a}$ and $\theta_{b}$, and one time delay $\tau_{c}$ by using two sensor arrays $\mathrm{Aa}$ and $\mathrm{Ab}$, and the sensor Sc, respectively. The pairs of $\theta_{a}$ and $\tau_{c}$, and $\theta_{b}$ and $\tau_{c}$ can be decided by TSaT-MUSIC. As shown in Figure 5, the position of the transmitter from Sc is described as:

$$
\begin{aligned}
& \left(x_{0}, y_{0}, z_{0}\right)= \\
& \quad\left(c \tau_{c} \cos \theta_{a}, c \tau_{c} \cos \theta_{b}, c \tau_{c} \sqrt{1-\left(\cos ^{2} \theta_{a}+\cos ^{2} \theta_{b}\right)}\right) .
\end{aligned}
$$

Hence, we can estimate the transmitter's position by using the TSaT-MUSIC algorithm.

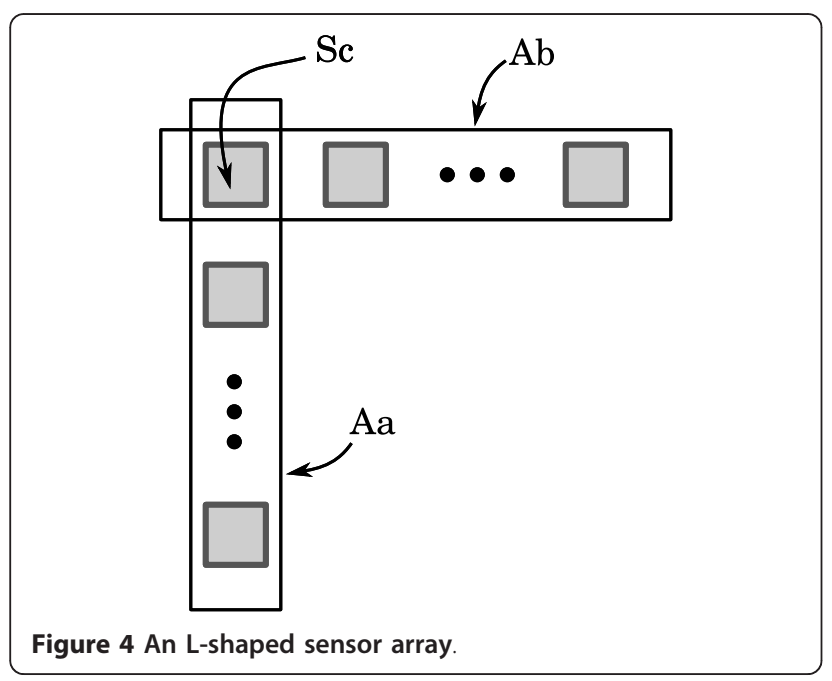

\subsection{Computational complexity}

The procedure of the TSaT-MUSIC algorithm includes one S-MUSIC calculation and two T-MUSIC calculations. The computational complexity of the S-MUSIC algorithm can be written as max $\left(\mathrm{O}\left(\mathrm{K}^{3}\right), \mathrm{O}\left(\mathrm{hK}^{2}\right)\right)$, where $h$ is the number of searches conducted along the DOA axis, $\mathrm{O}\left(\mathrm{K}^{3}\right)$ is the computational complexity of the eigenvalue decomposition of $R \boldsymbol{s s}$, and $\mathrm{O}\left(\mathrm{hK} \mathrm{K}^{2}\right)$ is that of the 1D spatial search. In the same way, the computational complexity of T-MUSIC can be expressed as $\max \left(\mathrm{O}\left(\mathrm{M}^{3}\right), \mathrm{O}\left(\mathrm{h}_{\mathrm{t}} \mathrm{M}^{2}\right)\right)$, where $h_{t}$ is the number of searches conducted along the time delay axis. Because $K$ is generally smaller than $M$, the computational complexity of the TSaT-MUSIC algorithm is given $\operatorname{bymax}(\mathrm{O}$ $\left.\left(\mathrm{M}^{3}\right), \mathrm{O}\left(\mathrm{h}_{\mathrm{t}} \mathrm{M}^{2}\right)\right)$.

On the other hand, the computational complexity of the 2D-MUSIC algorithm is $\max \left(\mathrm{O}\left((\mathrm{KM})^{3}\right), \mathrm{O}\left(\mathrm{hh}_{\mathrm{t}}\right.\right.$ $\left.(\mathrm{KM})^{2}\right)$ ) and that of the TST-MUSIC algorithm is max $\left(\mathrm{O}\left(\mathrm{LM}^{3}\right)\right.$, $\left.\mathrm{O}\left(\mathrm{Lh}_{\mathrm{t}} \mathrm{M}^{2}\right)\right)$. This means that the TSTMUSIC algorithm must perform the T-MUSIC calculation at least $L$ times. Consequently our proposed algorithm is theoretically faster than the existing method using the MUSIC algorithm.

\section{Simulations}

\subsection{Simulation setting}

We conducted two computer simulations using a PC (Dell Latitude D630, CPU: Intel(R) Core2Duo $2.60 \mathrm{GHz}$,

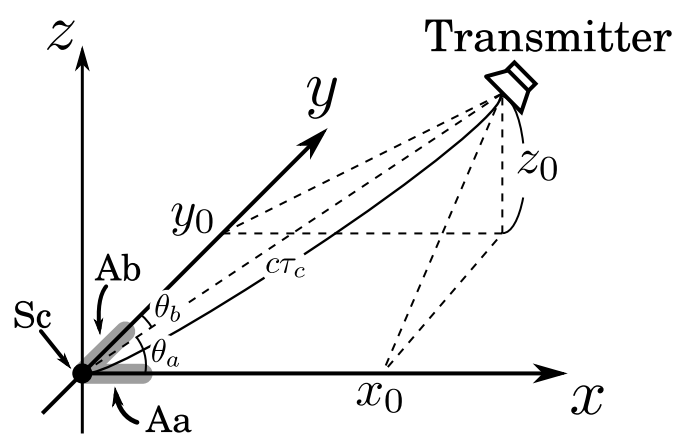

Figure $\mathbf{5}$ The position of the transmitter. 
Memory: 2.0 GB) and GNU Octave 3.0.1. The transmitted multicarrier ultrasonic wave has six subcarriers that are orthogonal to each other. The multicarrier wave $x(t)$ can be expressed as:

$$
x(t)=\sum_{i=0}^{N-1} a_{i} \sin \left\{2 \pi\left(f_{0}+i \Delta f\right) t+\phi_{i}\right\},
$$

where $N$ is the number of subcarriers (here, $N=6$ ), $f_{0}$ is the center frequency, $\Delta f$ is the interval of the subcarriers, $a_{i}$ is the amplitude of the $i$ th carrier, and $\varphi_{i}$ is its initial phase. In this simulation, all the subcarriers have the same amplitude, and their initial phases are given so that the transmitted wave has a peak amplitude at the half time of the transmission period $(0.5 \mathrm{~ms})$. The frequencies of the subcarriers are $34-39 \mathrm{kHz}$ (their interval was $1 \mathrm{kHz}$ ). Because the bandwidth of the wave was 5 $\mathrm{kHz}$, the theoretical time resolution was $0.2 \mathrm{~ms}$. In the simulations, DOA-delay estimations in the $2 \mathrm{D}$ space were conducted using a linear array sensor consisting of 16 receiver sensors at $5-\mathrm{mm}$ intervals. The SNR (Signal Noise Ratio) of the multicarrier wave is set to $20 \mathrm{~dB}$ by adding Gaussian noise.

As we plan to use the TSaT-MUSIC algorithm for rapid ultrasonic imaging by detecting reflectors, all sound sources transmit the same signal in this simulation. Therefore, receiving waves at a receiver array become coherent. To control the effect of the coherence, we use the spatial smoothing method [14] in SMUSIC and T-MUSIC algorithm, respectively. In 2D localization simulations, the number of sensors in a subarray $(K)$, the number of frequencies $(M)$, and the number of searches conducted along the time delay axis $\left(h_{t}\right)$ were set to 11,15 , and 1,000 , respectively.

From the principle of the TSaT-MUSIC algorithm as described in the previous section, its angular and range resolutions are same as those of the 1D S-MUSIC and T-MUSIC algorithms, respectively. Therefore, comparative evaluations between the proposed method and the TST-MUSIC algorithm are conducted through computer simulations shown in this section and experiments in real environments shown in the next section.

\subsection{Computational complexity and accuracy}

The DOA and delay values were set as shown in Table 1 by changing the number $(L)$ of incoming waves. The simulations of the TSaT-MUSIC and TST-MUSIC algorithms for each $L$ were conducted 1,000 times, and their

Table 1 The DOA and delay time values of incoming
waves
\begin{tabular}{lllllllllll}
\hline DOA [degrees] & -60 & -45 & -30 & -15 & 0 & 15 & 30 & 45 & 60 \\
Delay time [ms] & 0.6 & 1.2 & 1.8 & 2.4 & 3.0 & 3.6 & 4.2 & 4.8 & 5.4 \\
\hline
\end{tabular}

average computation times and values of the root mean square error (RMSE) were measured. Figure 6a shows the simulation results of the computation times. From the figure, we find that the TSaT-MUSIC algorithm can estimate DOAs and delays in almost a constant time. On the other hand, the computation time of the TSTMUSIC algorithm increases linearly as $L$ increases. This is consistent with the theory discussed in Section 3.3. Therefore, the computational complexity of the TSaTMUSIC algorithm is proved to be less than that of the TST-MUSIC algorithm. Figure $6 \mathrm{~b}$ shows the simulation results of the RMSE. These values were calculated by estimated positions of a sound source at $\left(-60^{\circ}, 0.6 \mathrm{~ms}\right)$. From the figure, we find that the values of RMSE using the TSaT-MUSIC algorithm is higher than that using TST-MUSIC algorithm regardless of the number of waves. However, all the RMSE values of the TSaTMUSIC algorithm are less than $6 \mathrm{~mm}$, which indicates the satisfactory level of accuracy.

\subsection{Robustness}

When positions of ultrasonic transmitters change, two or more candidate points become close to a path difference line. Figure 7 shows that two candidate points almost lie on a path difference line, when transmitters are placed at points $(125.7,713.0),(-939.5,341.9)$, and $(746.38,430.9)$ (unit: $\mathrm{mm})$. In order to investigate the robustness of the TSaT-MUSIC algorithm in such situations, simulations were conducted by setting SNRs between 0 and $50-\mathrm{dB}$ at $5-\mathrm{dB}$ intervals. Probabilities of selecting a true point were calculated through simulations conducted 100 times for each SNR as shown in Figure 8 . The figure demonstrates that the probabilities are 30 to $40 \%$ when the SNRs are lower than $30 \mathrm{~dB}$. However, when they are $30 \mathrm{~dB}$ or higher, the probabilities are almost $100 \%$. We are now investigating the reason why the performance of the proposed algorithm deteriorates when the SNR is lower than $30 \mathrm{~dB}$. At the moment, we suppose that it is due to the effects of the coherence between receiving signals. When the SNR is higher than $30 \mathrm{~dB}$, the effects of the coherence are controlled and the proposed algorithm retains higher probabilities of selecting true points.

\subsection{D localization simulations}

In this simulation, the L-shaped sensor array consisting of 15 receiver sensors at $5-\mathrm{mm}$ intervals was used. The same signals were transmitted from transmitters, and the spatial smoothing method was applied in the same way as in the $2 \mathrm{D}$ localization simulations. The SNR was set to $20 \mathrm{~dB}$. Because the L-shaped sensor array had 8 sensors on each side, the number of waves which could be classified was up to 4 . Hence, the number of transmitters was set to 3 . 
The transmitters were placed at points $\mathrm{Ta}(800,-300$, $400)$, Tb $(0,200,2,000)$, and Tc $(-900,-850,700)$ (unit: $\mathrm{mm})$. The L-shaped sensor was placed in the $x-y$ plane in the same way as in Figure 5. The average and standard deviations of the transmitters' positions were calculated for 25 simulations.

The results of the simulations are shown in Table 2. They show that the TSaT-MUSIC algorithm selected correct pairs of DOA and delay values. Differences between the true and estimated positions came from errors in the S-MUSIC or T-MUSIC algorithms.

\section{Experiments}

\subsection{Configuration of experimental system}

The configuration of the experimental system is shown in Figure 9. The signal processing board includes A/D converters and an operational amplifier. First, the PC receives the trigger signal that is sent by the signal processing board.

Next, the waveform generator (ADLINK DAQe-2501) creates signals that are sent to the ultrasonic transmitters (PIONEER PT-R4, Figure 10a) through the amplifier. The ultrasonic wave is transmitted from the transmitter to the receiver sensor array, where 15 ultrasonic sensors (SPM0204UD5 from Knowles, Figure 10b) are arranged in an L-shaped manner (the interval between sensors is $5 \mathrm{~mm}$ ) for 3D localization experiments. Finally, the signals received at the ultrasonic receiver are sent to the $\mathrm{PC}$ through the signal processing board. The parameters of the ultrasonic waves used in this experiment (such as frequencies and phases of subcarriers) were the same as those used in the computer simulations.

\subsection{D localization using TSaT-MUSIC}

3D localization experiments in real environments were conducted by using the TSaT-MUSIC algorithm to estimate the positions of three ultrasonic transmitters. These transmitters were set at the same positions as in the simulations in Section 4.

The measured average and standard deviation values of the ultrasonic transmitters' positions were obtained as shown in Table 3. All standard deviation values were less than $10 \mathrm{~mm}$, proving that the accuracy of the proposed algorithm was satisfactory for indoor 3D localization. The differences between the true positions and calculated average positions were larger in the real experiments than in the computer simulations. One reason for this was inaccurate placement of the transmitters and receivers. Sensor characteristics such as directivity might also affect the performance of the 3D localization. The other reasons for this performance deterioration in real experiments seemed related to the attenuation of the transmitted signals or multipath problems that were not taken into account in the simulations. To improve the accuracy of the proposed algorithm, intensive investigations through further experiments are necessary.

\subsection{Comparisons with TST-MUSIC}

Through experiments in real environment, we compared TSaT-MUSIC with TST-MUSIC in terms of RMSE values and computation times. Because TST-MUSIC algorithm is $2 \mathrm{D}$ localization algorithm, we conducted 2D localization experiment.

In the experiments, we used a linear sensor array including 16 receiver sensors arranged at 5-mm intervals. The other experimental settings were same as the $3 \mathrm{D}$ localization experiments described in this section. The localization measurements were taken 25 times. The transmitters were placed at points $\mathrm{Ta}(-300,300)$, $\mathrm{Tb}(0,1,000)$, and $\mathrm{Tc}(1,000,800)$ (unit: $\mathrm{mm})$, respectively. The measured values of RMSE are shown in Table 4. From this table, it is found that the accuracy (a)

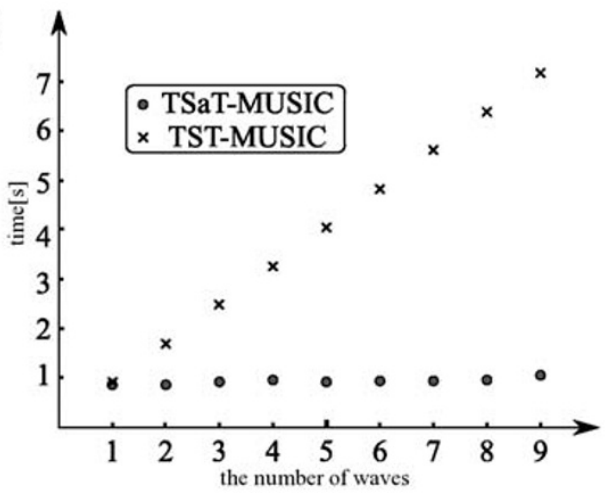

(b)

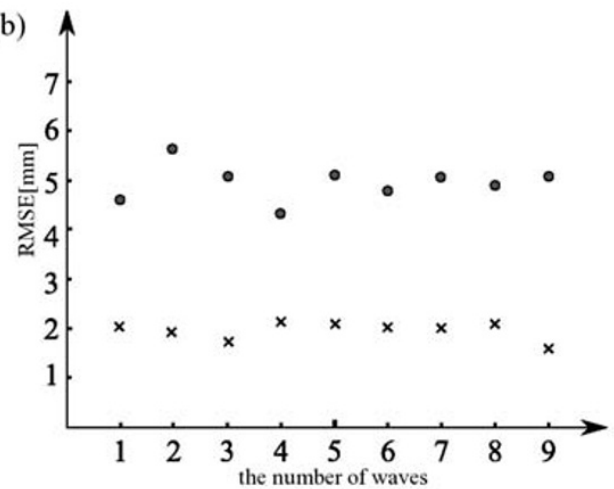

Figure 6 (a) Computation times of the TSaT-MUSIC and TST-MUSIC algorithms (b) RMSE of the TSaT-MUSIC and TST-MUSIC algorithms. 


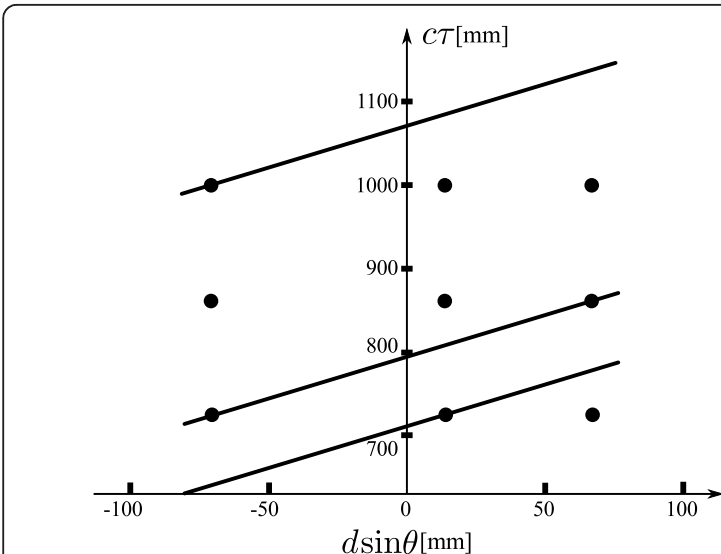

Figure 7 Path difference lines and candidate points

differences between TSaT-MUSIC and TST-MUSIC are not so remarkable. In comparison with the simulation results in Section 4, the RMSE values of TSaT-MUSIC and TST-MUSIC became worse. This deterioration was presumably caused by the same reasons as discussed in the 3D localization experiment. As for the computation time, it was confirmed that the TSaT-MUSIC algorithm was 1.8 times faster than the TST-MUSIC algorithm. Thus, the experiments indicated that TSaT-MUSIC was smaller than TST-MUSIC in their computational complexity and still retains the satisfactory level of localization accuracy.

\section{Conclusions and future work}

We have proposed a new DOA-delay estimation algorithm called the TSaT-MUSIC algorithm. The remarkable feature of the algorithm is its small computational complexity compared with existing algorithms based on the MUSIC algorithm. This feature was proved through computer simulations. Moreover, the 3D localization technique using the TSaT-MUSIC algorithm was verified to show its satisfactory accuracy using

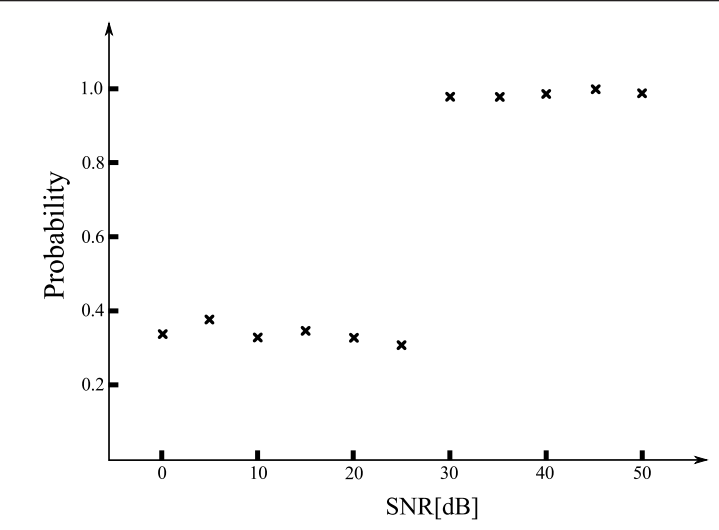

Figure 8 Probabilities of selecting true points.
Table 2 Results of the computer simulation (unit: $\mathbf{m m}$ )

\begin{tabular}{llccc}
\hline & & $\boldsymbol{x}$ & $\boldsymbol{y}$ & $\boldsymbol{z}$ \\
\hline \multirow{2}{*}{ Ta } & True position & 800.00 & -300.00 & 400.00 \\
& Average position & 799.16 & -310.17 & 393.58 \\
& Standard deviation & 1.12 & 3.84 & 4.34 \\
& True position & 0.00 & 200.00 & 2000.00 \\
Tb & Average position & 0.25 & 200.92 & 1999.70 \\
& Standard deviation & 3.66 & 5.51 & 0.66 \\
& True position & -900.00 & -850.00 & 700.00 \\
Tc & Average position & -905.48 & -837.14 & 708.40 \\
& Standard deviation & 2.50 & 6.56 & 8.37 \\
\hline
\end{tabular}

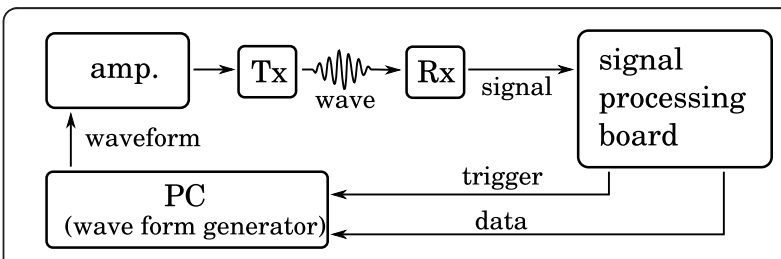

Figure 9 System configuration.

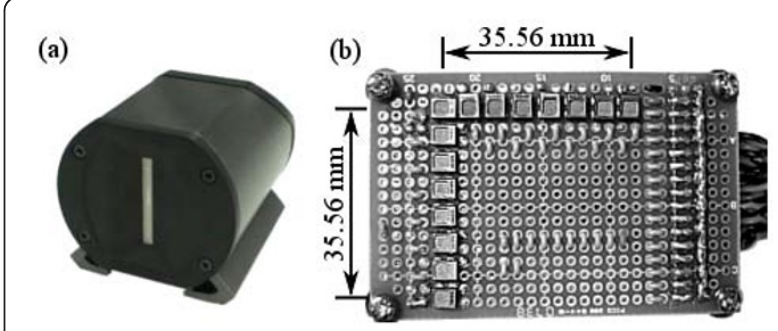

Figure 10 (a) Ultrasonic transmitter (b) ultrasonic receiver

computer simulations and experiments in real environments. There are several remaining issues to be investigated. Improving the performance of $3 \mathrm{D}$ localization using the proposed algorithm is one of the most important future tasks. Another important task is to improve the robustness of the TSaT-MUSIC algorithm

Table 3 Results of the experiment in real environments (unit: $\mathbf{m m}$ )

\begin{tabular}{llccc}
\hline & & $\boldsymbol{x}$ & $\boldsymbol{y}$ & $\boldsymbol{z}$ \\
\hline \multirow{2}{*}{$\mathrm{Ta}$} & True position & 800.00 & -300.00 & 400.00 \\
& Average position & 836.00 & -320.06 & 401.20 \\
& Standard deviation & 0.415 & 1.71 & 2.2 \\
& True position & 0.00 & 200.00 & 2000.00 \\
$\mathrm{~Tb}$ & Average position & -23.73 & 251.65 & 2036.57 \\
& Standard deviation & 2.91 & 1.19 & 8.40 \\
& True position & -900.00 & -850.00 & 700.00 \\
$\mathrm{Tc}$ & Average position & -918.74 & -867.48 & 702.68 \\
& Standard deviation & 0.47 & 2.36 & 3.02 \\
\hline
\end{tabular}


Table 4 Obtained values of RMSE (unit: $\mathbf{m m}$ )

\begin{tabular}{lll}
\hline & TSaT-MUSCI & TST-MUSIC \\
\hline $\mathrm{Ta}$ & 17.1 & 14.3 \\
$\mathrm{~Tb}$ & 21.1 & 28.3 \\
$\mathrm{Tc}$ & 81.1 & 75.0 \\
\hline
\end{tabular}

when a true point is not clearly identified due to placements of transmitters and low SNRs. We also plan to develop applications using our 3D localization technique, such as indoor positioning and robot navigation systems.

\section{Author details}

'Department of Electrical Engineering and Information Systems, School of Engineering, University of Tokyo 7-3-1 Hongo, Bunkyo-ku, Tokyo, 113-8656, Japan ${ }^{2}$ National Institute of Informatics, 2-1-2 Hitotsubashi, Chiyoda-ku, Tokyo, 101-8430, Japan

\section{Competing interests}

The authors declare that they have no competing interests.

Received: 9 November 2010 Accepted: 10 November 2011 Published: 10 November 2011

\section{References}

1. NB Priyantha, A Chakraborty, H Balakrishnan, The cricket location-support system, in MobiCom '00: Proceedings of the 6th annual international conference on Mobile computing and networking, ACM, New York, 32-43 (2000)

2. W Qiu, Application of the Location and Tracking System Based on Cricket, volume 106 of Communications in Computer and Information Science (Springer, Berlin, 2010)

3. OJ Woodman, RK Harle, Concurrent scheduling in the active bat location system, in Pervasive Computing and Communications Workshops (PER-COM Workshops), 2010 8th IEEE International Conference on, 431-437 (April 2010)

4. H Zhu, H Inubushi, N Takahashi, K Taniguchi, An ultrasonic 3D image sensor employing PN code, in Sensors 2006 5th IEEE Conference on 319-322 (Oct 2006)

5. K Nishihara, T Yamaguchi, H Hachiya, Position detection of small objects in indoor environments using coded acoustic signal. Acoust Sci Technol. 29(1), 15-20 (2008). doi:10.1250/ast.29.15

6. R Schmidt, Multiple emitter location and signal parameter estimation. IEEE Trans Antennas Propag. 34(3), 276-280 (1986). doi:10.1109/ TAP.1986.1143830

7. W Yong, Z Ping, L Lifang, W Linjing, J Qianqian, W Wei, An acoustic array imaging algorithm for $3 \mathrm{~d}$ display based on mimo and music location fusion, in Computer Application and System Modeling (ICCASM), 2010 International Conference on. 9, V9-107-V9-110 (2010)

8. Yasutaka Ogawa, Norihiro Hamaguchi, Kohzoh Ohshima, Kiyohiko Itoh, High-Resolution Analysis of Indoor Multipath Propagation Structure. IE-ICE TRANSACTIONS on Communications. E78-B(11), 1450-1457 (1995)

9. T Yasuhiko, O Yasutaka, O Takeo, High-resolution estimation of multipath propagation based on the 2D-MUSIC algorithm using time-domain signals. IEICE Trans Commun. 83(4), 407-415 (2000)

10. MC Vanderveen, Joint angle and delay estimation (JADE) for multipath signals arriving at an antenna array. IEEE Commun Lett. 1(12) (1997)

11. A Fujita, T Fukue, N Hamada, Both direction and time of arrival estimation by using beamforming and MUSIC algorithm for stepped FM array radar, in Circuits and Systems. 2004 MWSCAS '04. The 2004 47th Midwest Symposium on. 2, II-85-II-88 (July 2004)

12. YY Wang, JT Chen, WH Fang, TST-MUSIC for joint DOA-delay estimation. IEEE Trans Signal Process. 49(4), 721-729 (2001). doi:10.1109/78.912916

13. M Wax, T Kailath, Detection of signals by information theoretic criteria. IEEE Trans Acoust Speech Signal Process. 33(2), 387-392 (1985). doi:10.1109/ TASSP. 1985.1164557
14. TJ Shan, M Wax, T Kailath, On spatial smoothing for direction-of-arrival estimation of coherent signals. IEEE Trans Acoust Speech Signal Process. 33(4), 806-811 (1985). doi:10.1109/TASSP.1985.1164649

doi:10.1186/1687-6180-2011-101

Cite this article as: Mizutani et al.: TSaT-MUSIC: a novel algorithm for rapid and accurate ultrasonic 3D localization. EURASIP Journal on Advances in Signal Processing 2011 2011:101.

\section{Submit your manuscript to a SpringerOpen ${ }^{\mathcal{O}}$ journal and benefit from:}

- Convenient online submission

- Rigorous peer review

- Immediate publication on acceptance

- Open access: articles freely available online

- High visibility within the field

- Retaining the copyright to your article

Submit your next manuscript at $>$ springeropen.com 